\title{
Algumas velocidades de órbitas planetárias
}

\section{Some planetary orbits velocities}

\author{
Mylena Marins do Amaral \\ Universidade do Estado do Rio de Janeiro (UERJ), Faculdade de Formação de Professores \\ São Gonçalo, RJ, Brasil \\ http://orcid.org/0000-0001-7438-3970, mylena1579@hotmail.com
}

\begin{abstract}
Rosa María García Márquez
Universidade do Estado do Rio de Janeiro (UERJ), Faculdade de Formação de Professores

São Gonçalo, RJ, Brasil

http://orcid.org/0000-0003-3465-569X, rosagmarquez@yahoo.com.br
\end{abstract}

Jorge Corrêa de Araújo
Universidade do Estado do Rio de Janeiro (UERJ), Faculdade de Formação de Professores
São Gonçalo, RJ, Brasil
http://orcid.org/0000-0002-1015-6311, jcaraujo@iprj.uerj.br

\section{Informações do Artigo \\ Como citar este artigo \\ AMARAL, Mylena Marins do; MÁRQUEZ, \\ Rosa María García; ARAÚJO, Jorge Corrêa de. Algumas velocidades de órbitas \\ planetárias. REMAT: Revista Eletrônica da \\ Matemática, Bento Gonçalves, RS, v. 6, n. 1, \\ p. 01-14, jan. 2020. DOI: \\ https://doi.org/10.35819/remat2020v6i1id3512}

\section{Histórico do Artigo}

Submissão: 29 de junho de 2019.

Aceite: 06 de novembro de 2019.

\begin{abstract}
Resumo
No presente trabalho são apresentados dados coletados na literatura científica em relação ao afélio e ao periélio de alguns corpos celestes, e com base na primeira lei de Kepler, são determinadas as equações elípticas que descrevem suas trajetórias ao redor do Sol. Utilizando o binômio de Newton, e conceitos básicos de conservação de energia e do momento angular de um sistema físico, pode ser obtida a velocidade máxima orbital de cada corpo celeste aqui analisado em função da excentricidade de sua órbita elíptica e de seu eixo. Obtido o tempo orbital de cada um desses corpos celestes e o comprimento de suas trajetórias em torno do Sol, sua velocidade média orbital foi calculada usando a cinemática do movimento. Os resultados obtidos usando essa metodologia simplificada encontram-se em boa concordância com os divulgados pelos astrônomos.
\end{abstract}

\begin{abstract}
In the present work are presented data collected in the scientific literature regarding the aphelion and the perihelion of some celestial bodies and based on the first law of Kepler are determined the elliptic equations that describe their trajectories around the Sun. Using Newtons's binomial and basic concepts of energy conservation and angular momentum of a system, can be obtained the maximum velocity of the each celestial body here analyzed as a function of the eccentricity of the elliptical orbit and it axis. Obtaining the orbital time of each of these celestial bodies and the length of their trajectories around the Sun, their average orbital velocity was calculated using the kinematics of the motion. The results obtained using this simplified methodology are good in agreement with those published by astronomers.
\end{abstract}

\section{Introdução}

Olhar o céu durante uma noite sem nuvens é um belo espetáculo natural. Mesmo aqueles que não têm grande interesse pela astronomia, em noites como essas, não ficam indiferentes à beleza da cena. Segundo Nogueira (2009), desde a pré-história, o homem movido pela 
curiosidade observou e estudou os padrões dos movimentos dos astros, passando a fazer uso desse conhecimento para previsões astronômicas com objetivos científicos, bem como, analisar seus efeitos sobre o mundo em que vivemos.

Embora, a humanidade tenha desenvolvido métodos científicos sofisticados para realizar previsões astronômicas como, por exemplo, medir o tempo e orientar-se no espaço, muitos dos métodos mais rudimentares são ainda utilizados por conta de sua simplicidade e por fornecerem resultados aproximados, principalmente na observação de estrelas. A simples observação da Estrela Polar para quem se encontra no Hemisfério Norte, e da constelação Cruzeiro do Sul, para quem se encontra no Hemisfério Sul, permite saber a localização dos pontos cardeais os quais são utilizados na orientação espacial (RAMOS, 2006).

As constelações são definidas como "agrupamentos aparentes de estrelas" (LONGHINI, 2009), e fato bastante curioso acerca das constelações é que, para um observador na Terra, as estrelas que as compõem parecem bem próximas, mas na realidade, estão a distâncias muito grandes umas das outras, medidas em anos-luz. A Figura 1 é devida a Longhini (2009), e mostra a constelação mais famosa do Hemisfério Sul conhecida como Cruzeiro do Sul. Na esquerda da Figura 1, as estrelas parecem agrupadas, mas de acordo com Mourão (1989, apud LONGHINI, 2009) as respectivas distâncias entre as estrelas de Magalhães, Mimosa, Rubídea, Pálida e Intrometida ao planeta Terra são: 359, 424, 88, 257 e 58 anos-luz, sendo que a menor distância entre duas delas é de aproximadamente 30 anos-luz.

Figura 1 - Posição das estrelas da constelação Cruzeiro do Sul.

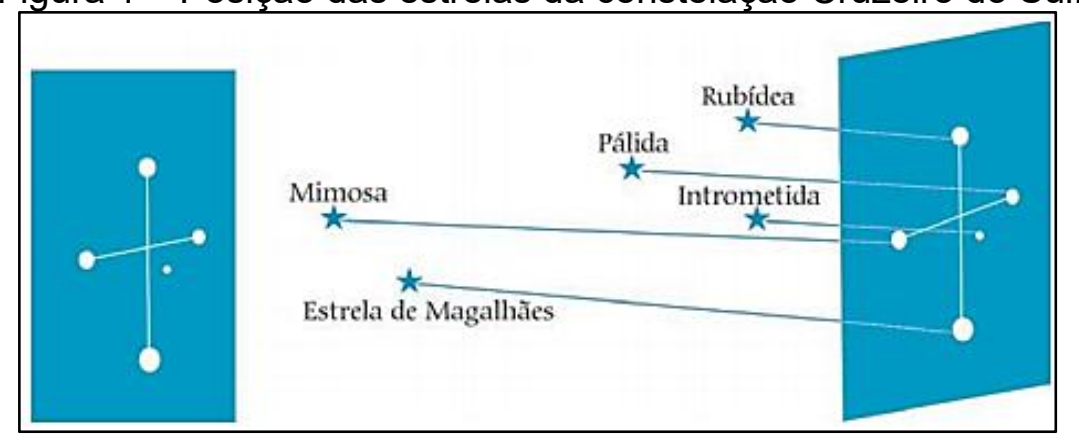

Fonte: Longhini (p. 27, 2009).

Para compreender melhor as distâncias em que essas estrelas se encontram de nós, consideremos o planeta Vênus, que é o planeta que está mais próximo da Terra, e mesmo assim, encontra-se a uma distância média de 41 milhões de quilômetros (WILLIAMS, 2016). Sabendo-se que um ano-luz equivale aproximadamente a $9,461 \times 10^{12} \mathrm{~km}$, então a distância média de Vênus à Terra é de $4,334 \times 10^{-6}$ anos-luz. Portanto, a distância entre Vênus e a Terra é irrisória em comparação às distâncias entre as estrelas da constelação Cruzeiro do Sul.

O objetivo desse trabalho é apresentar de forma resumida parte da história da astronomia e calcular as velocidades orbitais de cinco planetas do sistema solar, usando somente as Leis de Kepler, cinemática e gravitação universal. 


\section{Um pouco de História sobre a Astronomia}

Desde a pré-história, os seres humanos foram capazes de perceber os padrões de movimento dos astros e, com isso, passaram a diferenciar os dias e as estações do ano (Nogueira, 2009). Um dos primeiros astrônomos conhecidos foi o grego Eudoxo de Cnido (408 a.C.-355 a.C.), a quem foi atribuída a divisão do ano solar como é utilizada hoje em dia. Eudoxo também postulou que a Terra era um planeta esférico fixo, sendo o centro do universo, envolta de 27 esferas. Posteriormente, o grego Cláudio Ptolomeu (90-168) ficou famoso com a Teoria Geocentrista, a qual considerava a Terra como o centro do universo com os demais planetas girando em sua volta com trajetórias circulares, enquanto o centro desta órbita descrevia outra trajetória (epiciclos). O avanço científico ficou estagnado por quase 15 séculos sem que essa teoria fosse questionada (Nogueira, 2009).

Segundo Nogueira (2009), somente em 1543, o matemático e astrônomo polonês Nicolau Copérnico defendeu a teoria heliocentrista. Nessa teoria, o Sol é o centro do universo com os planetas do sistema solar orbitando em sua volta. A teoria do heliocentrismo marcou o começo de uma nova era científica.

Ainda no século XVI, o físico e astrônomo italiano Galileu Galilei (1564-1642) comprovou o sistema heliocêntrico de Copérnico. Galileu também fez grandes contribuições à Astronomia sobre as manchas solares, montanhas da Lua, fases de Vênus, anéis de Saturno, satélites de Júpiter e as estrelas da via láctea (Mendonça, 2011). Paralelamente, o responsável por descartar de vez a teoria geocentrista, foi o matemático e astrônomo alemão Johannes Kepler (1571-1630), tomando como base as observações de Tycho Brake (1546-1601) (CANALLE, 2003), que percebeu que o modelo copernicano funcionava perfeitamente, ou seja, as órbitas planetárias não eram circulares, mas sim elípticas, sendo o Sol um de seus focos (Nogueira, 2009). Kepler deixou como legado três importantes leis sobre o movimento dos planetas conhecidas como as três leis de Kepler:

$1^{\text {a }}$ Lei: Todos os planetas se deslocam ao redor do Sol descrevendo órbitas elípticas. O Sol é um dos focos da elipse.

$2^{\mathrm{a}}$ Lei: O raio vetor que une um planeta e o Sol, percorre áreas iguais em tempos iguais.

$3^{a}$ Lei: Para qualquer planeta, o quadrado de seu período orbital é diretamente proporcional ao cubo do comprimento do semieixo maior de sua órbita.

Alguns anos depois, coube ao físico, matemático e astrônomo inglês, Isaac Newton (16431727), entre outros feitos notáveis, postular a "Lei da Gravitação Universal" que possibilitou explicar fenômenos que ocorrem tanto na superfície terrestre como no Espaço Sideral. Essa lei só foi contestada no início do século XX por Albert Einstein no seu tratado sobre a Teoria da Relatividade Geral, na qual considerava a gravidade exercida não como uma força entre corpos de grande massa, mas sim através do encurvamento do espaço a sua volta. Em 1917, Einstein aplicou suas equações para a teoria do universo e concluiu que ele deveria estar em expansão ou 
contração. Entretanto, havia o consenso na época que o cosmos estava estático, o que levou a Einstein a acrescentar em suas equações a constante cosmológica para atender a essa condição (NOGUEIRA, 2009).

Com base na Teoria da Relatividade Geral e nas equações de campo de Einstein, em 1929, o astrônomo americano Edwin Hubble (1889-1953), descobriu que as constelações se distanciam entre si, e quanto mais longe a constelação, mais rápido ocorre o afastamento, concluindo, desse modo, que o universo está em expansão, contrariando a constante cosmológica de Einstein (NOGUEIRA, 2009).

Com o desenvolvimento dos telescópios e radiotelescópios, bem como outros instrumentos de grande precisão como os telescópios espaciais, novos corpos celestes são conhecidos e mais informações sobre a natureza do nosso universo são encontradas. A energia escura, por exemplo, que é a força responsável pela aceleração da expansão do universo, foi descoberta graças à tecnologia empregada no telescópio espacial Hubble em órbita desde 1990 (SATO, 2009). Muitos astrônomos empenharam-se para que descobertas importantes como essa ocorressem e pudéssemos compreender melhor o universo, como por exemplo Tycho Brahe (1546-1601), Christiaan Huygens (1629-1695), Edmond Halley (1656-1742), Giovanni Cassini (1625-1712), Edwin Hubble (1889-1953), Arno Penzias (1933 --), Robert Wilson (1936 --), Marcelo Gleiser (1959 --), Joan Feynman (1927--), Stephen Hawking (1942-2018), Michael E. Brown (1965 --), entre outros (COSTA, 2006; SILVA, 2016).

\section{O Periélio e o Afélio}

Chama-se afélio o ponto em que um planeta está mais afastado do Sol, enquanto o periélio é o ponto da órbita em que um corpo celeste está mais perto do Sol (ESQUEF; RIBEIRO, 2012). Denotemos por A a distância entre o afélio e o Sol e por P a distância entre o periélio e o Sol, conforme pode ser visto na Figura 2.

Figura 2 - Representação aumentada da trajetória elíptica mostrando a posição do planeta e do afélio e periélio, e a trajetória de um corpo celeste ao redor do Sol.

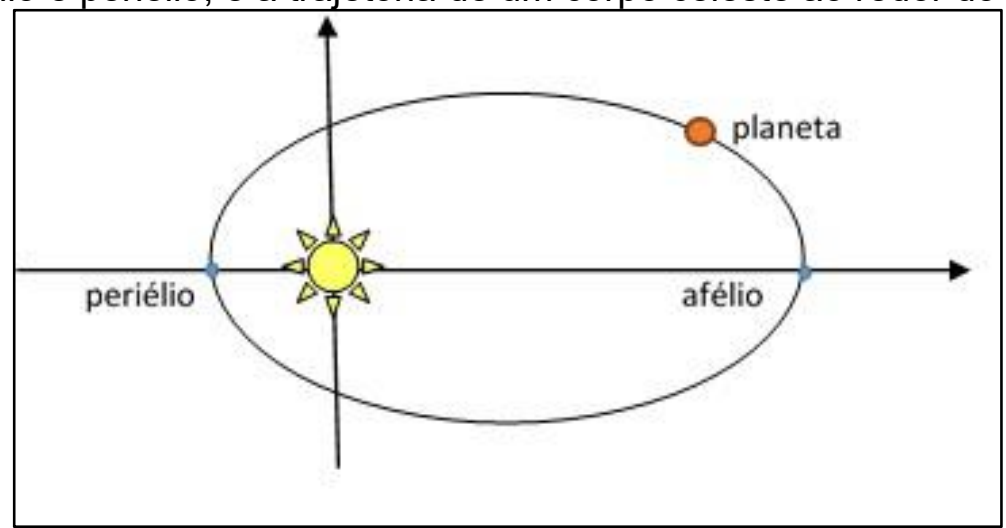

Fonte: Elaboração dos autores (2019). 


\section{4. Órbitas Elípticas}

Nessa seção apresenta-se a equação da elipse em função das distâncias $A$ e $P$, onde A é a máxima distância do planeta ao Sol, enquanto $P$ é a menor distância do planeta ao Sol (ESQUEF; RIBEIRO, 2012).

Figura 3 - Representação do posicionamento dos eixos coordenados.

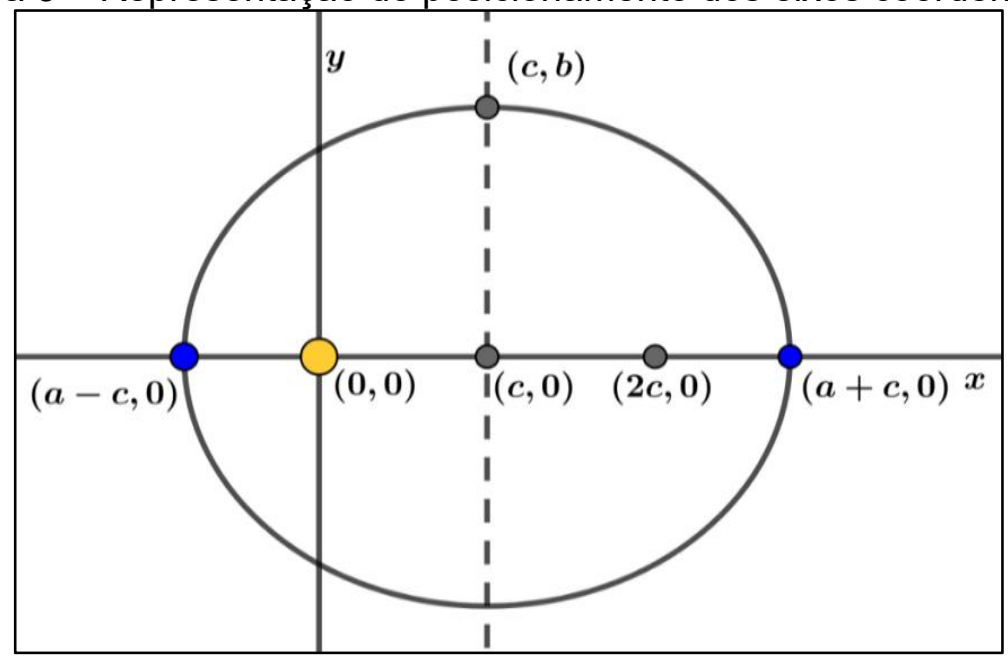

Fonte: Elaboração dos autores (2019).

A equação da elipse exibida na Figura 3 pode ser descrita no sistema cartesiano como

$$
\frac{(x-c)^{2}}{a^{2}}+\frac{y^{2}}{b^{2}}=1
$$

onde $(\mathrm{c}, 0)$ são as coordenadas do centro da elipse, a representa o semieixo maior enquanto $b$ o semieixo menor. As distâncias entre o afélio ao Sol e o periélio ao Sol, são determinadas e denotadas por

$$
\begin{aligned}
& A=\|(0,0)-(a+c, 0)\|=a+c \\
& P=\|(0,0)-(a-c, 0)\|=a-c .
\end{aligned}
$$

Das equações (2) e (3) obtemos os valores do semieixo maior e a metade da distância focal em função das distâncias $A$ e $P$

$$
a=\frac{A+P}{2} \text { e } c=\frac{A-P}{2} .
$$

Uma relação fundamental da elipse é $a^{2}=b^{2}+c^{2}$, então $b^{2}=\left(\frac{A+P}{2}\right)^{2}-\left(\frac{A-P}{2}\right)^{2}$, ou equivalentemente à

$$
b^{2}=A . P .
$$

Substituindo os valores obtidos das equações (4) e (5) na equação (1), obtemos a equação das trajetórias elípticas em função de $A$ e $P$ sendo dada por 


$$
\frac{\left(x-\frac{A-P}{2}\right)^{2}}{\left(\frac{A+P}{2}\right)^{2}}+\frac{Y^{2}}{A \cdot P}=1 .
$$

Na seção 5, apresenta-se a Tabela 1 contendo os valores de A e $\mathrm{P}$ para alguns planetas do sistema solar. Substituindo os valores da Tabela 1 na equação (6), é possível determinar a equação da trajetória elíptica de cada um dos planetas ao redor do Sol. A equação cartesiana da órbita elíptica de cada um dos planetas do sistema solar pode ser encontrada em Esquef e Ribeiro (2012).

Sabendo-se que a excentricidade de uma elipse é dada por $e=\frac{c}{a}$; a partir da equação (4) obtém-se a excentricidade em função dos valores $A$ e $P$

$$
e=\frac{A-P}{A+P} \text {. }
$$

\section{Comprimento de Arco}

O comprimento de arco de uma curva $\beta$ (GUIDORIZZI, 2002), é dada na forma paramétrica por

$$
L(\beta)=\int_{0}^{2 \pi}\left\|\beta^{\prime}(t)\right\| d t
$$

Portanto, uma parametrização da elipse dada pela equação (1) pode ser colocada na forma

$$
\beta(t)=(a \operatorname{sen}(t), c+b \cos (t)) ; t \in[0,2 \pi] .
$$

Das equações (8) e (9) o comprimento de arco é dado pela integral elíptica completa de segunda espécie (HASSER; LA SALLE; SULLIVAN, 1990)

$$
L(\beta)=\int_{0}^{2 \pi} \sqrt{a^{2} \cos ^{2}(t)+b^{2} \operatorname{sen}^{2}(t)} d t .
$$

Considerando que $b^{2}=a^{2}-c^{2}$, e que a excentricidade $e$ é dada pela relação $e=\frac{c}{a}$, então a equação (10) pode ser reescrita na forma

$$
L(\beta)=4 a \int_{0}^{\frac{\pi}{2}} \sqrt{1-e^{2} \operatorname{sen}^{2}(t)} d t .
$$

Observe que se a excentricidade $e$ for nula, decorre da equação (11) que $L(\beta)=$ $4 a \int_{0}^{\frac{\pi}{2}} 1 d t=2 \pi a$, o que corresponde ao comprimento de uma circunferência de raio $a$.

Se $0<e<1$, a integral dada na equação (11) não pode ser expressa de forma finita por meio de funções elementares. Usando o binômio de Newton podemos expressar o integrando da equação (11) como uma aproximação de uma série finita de potências de fácil integração.

Denotemos por $K=-e^{2} \operatorname{sen}^{2}(t)$. Assim, o integrando $(1+K)^{\frac{1}{2}}$ da equação (11) pode ser escrito como 


$$
(1+K)^{\frac{1}{2}}=1+\frac{1}{2} k-\frac{1}{8} k^{2}+\frac{1}{16} k^{3}-\frac{5}{128} k^{4}-\frac{7}{256} k^{5}+\ldots
$$

Para obter um valor numérico da equação (11), vamos a considerar apenas os quatro primeiros termos do binômio de Newton da equação (12). Assim,

$$
(1+K)^{\frac{1}{2}} \approx 1+\frac{1}{2} k-\frac{1}{8} k^{2}+\frac{1}{16} k^{3} .
$$

Substituindo na equação (13) a variável $K=-e^{2} \operatorname{sen}^{2}(\mathrm{t})$, tem-se que

$$
\left(1-e^{2} \operatorname{sen}^{2}(\mathrm{t})^{\frac{1}{2}} \approx 1-\frac{1}{2} e^{2} \operatorname{sen}^{2}(\mathrm{t})-\frac{1}{8} e^{4} \operatorname{sen}^{4}(\mathrm{t})-\frac{1}{16} e^{6} \operatorname{sen}^{6}(\mathrm{t}) .\right.
$$

Portanto, o comprimento da curva $\beta$, dada pela equação (11), pode ser aproximado por

$$
L(\beta)=4 a \int_{0}^{\frac{\pi}{2}} \sqrt{1-e^{2} \operatorname{sen}^{2}(t)} d t \approx 4 a \int_{0}^{\frac{\pi}{2}}\left(1-\frac{1}{2} e^{2} \operatorname{sen}^{2}(\mathrm{t})-\frac{1}{8} e^{4} \operatorname{sen}^{4}(\mathrm{t})-\frac{1}{16} e^{6} \operatorname{sen}^{6}(\mathrm{t})\right) \mathrm{dt} .
$$

Integrando os termos da direita da equação (15) e após simplificações algébricas, tem-se o valor aproximado do comprimento de arco em função da excentricidade e o semieixo maior,

$$
L(\beta) \approx \pi a\left(2-\frac{1}{2} e^{2}-\frac{3}{32} e^{4}-\frac{5}{128} e^{8}\right) .
$$

Como era de se esperar, para valores de $e$ muito pequenos na equação (16) tem-se $L(\beta) \approx$ $2 \pi a$.

\section{Lei Gravitacional de Newton e Velocidade Orbital}

A lei gravitacional de Newton afirma que os corpos celestes estão submetidos a uma força gravitacional que é diretamente proporcional às massas dos corpos em interação e é inversamente proporcional ao quadrado da distância entre eles (SILVA, 2001), isto é,

$$
F=\frac{G M m}{r^{2}}
$$

onde

$F$ : representa a força gravitacional entre dois objetos;

$M$ : massa do corpo principal;

$M$ : massa do corpo secundário;

$m$ : distância entre os centros de massa dos objetos;

$G$ :constante universal da gravitação.

Seja $m$ a massa de um planeta em movimento circular uniforme ao redor do Sol. A força centrípeta $F_{c}$, isto é, a força radial aplicada ao planeta e dirigida para o centro de sua trajetória, tem sua magnitude dada por (HALLIDAY; RESNICK; WALKER, 2002)

$$
F_{c}=\frac{m v^{2}}{r}
$$


onde $v$ é a velocidade orbital do planeta e $r$ o raio da circunferência. Como a força gravitacional, nesse caso, é igual à força centrípeta, então ao igualarmos as equações (17) e (18), obtemos a velocidade orbital dada por

$$
v=\sqrt{\frac{G M}{r}} .
$$

Sejam $M$ a massa do Sol e m a massa de um planeta em órbita elíptica em torno do Sol; podemos obter as velocidades orbitais máxima e mínima por meio da Lei da Gravitação Universal.

A Figura 4 mostra um planeta localizado em um ponto $Q$ arbitrário com trajetória elíptica e o Sol localizado no foco $F_{2}$. Também podem ser vistas as distâncias entre o periélio e o afélio.

\section{Figura 4 - Elipse com focos $F_{1}$ e $F_{2}$, e $L$ é uma das retas diretrizes.}

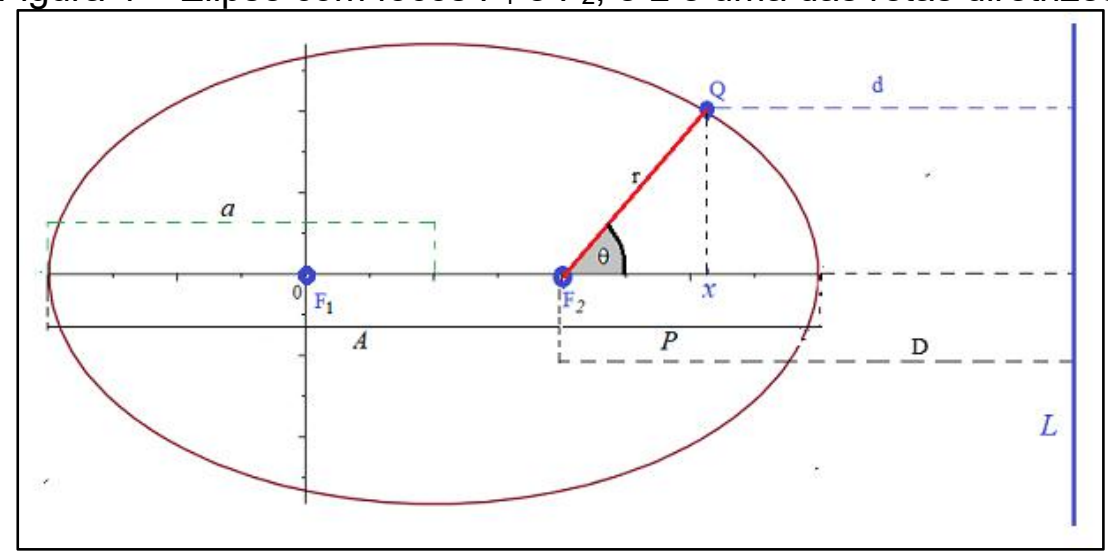

Fonte: Elaboração dos autores (2019).

Da Figura 4 obtém-se a relação com o semieixo maior dada por

$$
A+P=2 a \text {. }
$$

Seja $L$ a reta diretriz direita correspondente ao foco $F_{2}$ (reta perpendicular ao eixo focal). Denotando por $r=\operatorname{dist}\left(Q, F_{2}\right)$ e $d=\operatorname{dist}(Q, L)$, então por definição da elipse (VENERO, 1995) tem-se,

$$
\frac{r}{d}=\frac{\operatorname{dist}\left(Q, F_{2}\right)}{\operatorname{dist}(Q, L)}=e
$$

onde $e$ é a excentricidade $e<1$. Logo, da equação (21) segue que

$$
r=e d
$$

onde $d=D-x$ (Figura 4).

O ponto $Q$ escrito em coordenadas polares pode ser colocado na forma

$$
Q=(x, y)=(r \cos \theta, r \operatorname{sen} \theta) .
$$

Da equação (22) e da equação (23), a distância $r$ em coordenada polares pode ser escrita como

$$
r=e(D-r \cos \theta)
$$

ou ainda por 


$$
r(\theta)=\frac{e D}{1+e \cos \theta} .
$$

Ao substituir na equação (25) o valor de $\theta$ por 0 e por $\pi$, temos respectivamente

$$
r(0)=\frac{e D}{1+e} \text { e } r(\pi)=\frac{e D}{1-e}
$$

Observe que $r(0)+r(\pi)=2 a, r(0)=\operatorname{Pe} r(\pi)=A$. Somando as duas expressões obtidas em (26) temos

$$
\frac{e D}{1+e}+\frac{e D}{1-e}=2 a
$$

de onde obtemos $D$,

$$
D=\frac{a\left(1-e^{2}\right)}{e} .
$$

Substituindo o valor de $D$ encontrado na equação (28) e utilizando a equação (25), tem-se

$$
r(\theta)=\frac{a\left(1-e^{2}\right)}{1+e \cos \theta}
$$

Assim,

$$
P=r(0)=a(1-e) e A=r(\pi)=a(1+e)
$$

Por outro lado, sabemos que a conservação de energia mecânica total $E$, em qualquer ponto da órbita, é igual à soma da energia cinética $E_{c}$ com a energia potencial gravitacional $E_{p}$, e é constante, já que o campo gravitacional é conservativo (HALLIDAY; RESNICK; WALKER, 2002). Em termos de equação a energia mecânica total $E$ é dada por

$$
E=E_{c}+E_{p}=\text { const. }
$$

Assim,

$$
E=\frac{m v^{2}}{2}-\frac{G M m}{r}
$$

Seja $\varepsilon$ a energia orbital específica que é definida como a energia total da partícula por unidade de massa em órbita (ALRIGA, 2019). Multiplicando a equação (32) pelo inverso da massa do corpo, resulta

$$
\varepsilon=\frac{v_{A}^{2}}{2}-\frac{G M}{A} \text { e } \varepsilon=\frac{v_{P}^{2}}{2}-\frac{G M}{P},
$$

onde $v_{A}$ e $v_{P}$ representam respectivamente a velocidade máxima e mínima, quando o corpo se encontra no afélio ou no periélio.

Pela conservação do momento angular (HALLIDAY; RESNICK; WALKER, 2002) podemos escrever

$$
P m v_{P}=A m v_{A}
$$


Da equação (34), obtemos

$$
v_{P}=\frac{A v_{a}}{P} .
$$

Subtraindo as equações dadas em (33), temos

$$
\frac{v_{A}^{2}}{2}-\frac{v_{P}^{2}}{2}=\frac{G M}{A}-\frac{G M}{P} .
$$

Das equações (35) e (36), pode-se estabelecer a igualdade

$$
\frac{v_{A}^{2}}{P^{2}}\left(P^{2}-A^{2}\right)=\frac{G M}{A P}(P-A) \text {. }
$$

Da equação (30) e da equação (37) obtemos a velocidade orbital em função da excentricidade $e$ e do semieixo maior $a$, dada por

$$
v_{A}=\sqrt{\frac{2 G M P}{A(P+A)}}=\sqrt{\frac{2 G M a(1-e)}{a(1+e)(2 a)}} .
$$

Simplificando a equação (38), a velocidade mínima de um corpo orbitando ao redor do Sol pode ser expressa por

$$
v_{A}=\sqrt{\frac{G M}{a}\left(\frac{(1-e)}{(1+e)}\right)} .
$$

De forma análoga, a velocidade máxima do corpo orbitando ao redor do Sol (ALRIGA, 2019) pode ser escrita como

$$
v_{P}=\sqrt{\frac{G M}{a}\left(\frac{(1+e)}{(1-e)}\right)} .
$$

\section{Cálculo do Comprimento da Trajetória e da Velocidade Orbital}

Os planetas considerados nesse estudo com respeito as suas velocidades orbitais e comprimento de suas trajetórias são Vênus, Terra, Marte, Júpiter e Saturno. Para isso, são utilizadas simplesmente as equações (16) e (40). Além disso, os dados do afélio e do periélio podem ser vistos na Tabela 1. A constante de gravitação é $G=6,67408 \times 10^{-11} \mathrm{~m}^{3} / \mathrm{kg} \cdot \mathrm{s}^{2}$ (ou $\mathrm{Nm}^{2} / \mathrm{kg}^{2}$ ) e a massa do Sol é $\mathrm{M}=1,989 \times 10^{30} \mathrm{~kg}$ (ASIMOV, 1991).

Um exemplo utilizando essa metodologia pode ser aplicado ao planeta Vênus. 
Tabela 1 - Dados do afélio e periélio de alguns planetas do sistema solar.

\begin{tabular}{ccccc}
\hline Corpos Celestes & $A$ & $P$ & $A$ & $P$ \\
& $(U . A .)^{1}$ & $(U . A)$. & $(\mathrm{km})$ & $(\mathrm{km})$ \\
\hline Vênus - Sol & 0,72822 & 0,71846 & 108.940 .000 & 107.480 .000 \\
Terra - Sol & 1,01673 & 0,98324 & 152.100 .000 & 147.098 .000 \\
Marte - Sol & 1,66599 & 1,38117 & 249.230 .000 & 206.620 .000 \\
Júpiter - Sol & 5,45877 & 4,95007 & 816.620 .000 & 740.520 .000 \\
Saturno - Sol & 10,12381 & 9,04124 & 1.514 .500 .000 & 1.352 .550 .000 \\
\hline
\end{tabular}

Fonte: NASA (2019).

\subsection{Planeta Vênus e a Estrela Sol}

Considerando os dados na Tabela 1 de $A$ e $P$ para o planeta Vênus orbitando ao redor do Sol, pode-se obter da equação (4) o valor do semieixo maior dado por

$$
a=\frac{A+P}{2}=\frac{0,7288+0,71846}{2}=0,72334 \text { U.A. }
$$

Enquanto a excentricidade $e$ pode ser obtida da equação (7) em termos de $A$ e $P$ como

$$
e=\frac{A-P}{A+P}=\frac{0,7288-0,71846}{0,7288+0,71846}=0,006746 .
$$

Substituindo os dados obtidos em (41) e (42) na equação (16), obtemos o comprimento aproximado da trajetória elíptica que percorre o planeta Vênus em torno do Sol

$$
L(\beta) \approx \pi a\left(2-\frac{1}{2} e^{2}-\frac{3}{32} e^{4}-\frac{5}{128} e^{6}\right) \approx 4,544827 \text { U.A. }
$$

Sabendo que uma unidade astronômica (1 U.A.) equivale aproximadamente a 150 milhões de $\mathrm{km}$, então o comprimento da órbita elíptica de Vênus ao redor do Sol em $\mathrm{km}$ é

$$
L(\beta) \approx(4,544822)(149597870,7) \approx 6798957 \times 10^{8} \mathrm{Km} .
$$

Portanto, o planeta Vênus percorre, após uma órbita completa, cerca de $6,798957 \times$ $10^{8} \mathrm{~km}$ de distância.

Por outro lado, uma órbita completa do planeta Vênus em torno do Sol demora 224,695 dias (NASA, 2019). Desse modo, considerando o comprimento da órbita elíptica deste planeta pode-se estimar por meio da equação (44) a velocidade orbital média dada pela conhecida equação da cinemática:

$$
\text { espaço }=\text { velocidade } \times \text { tempo }
$$

Assim, a velocidade orbital média do planeta Vênus em torno do Sol é dada por

$$
\text { velocidade }=\frac{\text { espaço }}{\text { tempo }}=\frac{\Delta s}{\Delta t} \text {. }
$$

\footnotetext{
1 U.A. (Unidade astronômica) é uma unidade de distância, definida como a distância media entre a Terra e o Sol. Em 2012, a União Astronômica Internacional definiu o valor constante, igual 149.597.870,700 km, que é aproximadamente 150 milhões de km (SIMÕES, 2013).
} 
Substituindo os dados obtidos do comprimento de trajetória (44) e o tempo de duração na equação (46), obtemos a velocidade média

$$
\text { velocidade }=\frac{6,798957 \times 10^{8}}{224,695 \times 24 \times 60 \times 60}=35,02855 \frac{\mathrm{km}}{\mathrm{s}} .
$$

Utilizando a equação (40), pode-se estimar a velocidade máxima orbital do planeta Vênus como

$$
v_{P}=\sqrt{\frac{G M(1+e)}{a(1-e)}} \approx \sqrt{\frac{6,67408 \times 1,989 \times 10^{19}}{0,723339 \times 149597870,7 \times 10^{3}}\left(\frac{1+0,006746}{1-0,006746}\right)} \approx 35252,676 \mathrm{~m} / \mathrm{s} .
$$

Portanto a velocidade máxima de translação do planeta Vênus é $35,2527 \mathrm{~km} / \mathrm{s}$. Resultado em boa concordância com o valor obtido com a equação (47).

\subsection{Resultados Obtidos}

De forma análoga à realizada na seção 7.1 , foram calculadas as velocidades dos demais planetas; os resultados podem ser vistos nas Tabelas 2 e 3.

Tabela 2 - Dados do afélio, periélio, excentricidade e comprimento aproximado das órbitas elípticas, usando a equação (16).

\begin{tabular}{ccccc}
\hline Corpos Celestes & $\begin{array}{c}\text { Semieixo } \\
\text { Maior } \\
a=\frac{A+P}{2}\end{array}$ & $\begin{array}{c}\text { Excentricidade } \\
e=\frac{A-P}{A+P}\end{array}$ & $\begin{array}{c}\text { Comprimento } \\
\text { aproximado em } \\
\text { U.A. }\end{array}$ & $\begin{array}{c}\text { Comprimento } \\
\text { aprox. em } \mathbf{k m}\end{array}$ \\
\hline Vênus - Sol & 0,723339 & 0,006746 & 4,544827 & $6,798965 \times 10^{8}$ \\
Terra - Sol & 0,999985 & 0,016745 & 6,282651 & $9,398712 \times 10^{8}$ \\
Marte - Sol & 1,523585 & 0,093472 & 9,552060 & $1,428969 \times 10^{9}$ \\
Júpiter - Sol & 5,204419 & 0,048872 & 32,680818 & $4,888980 \times 10^{9}$ \\
Saturno - Sol & 0,723339 & 0,006746 & 4,544827 & $6,798965 \times 10^{8}$ \\
\hline
\end{tabular}

Fonte: Valores do semieixo maior e da excentricidade disponíveis em Fonte: NASA (2019).

Por meio de conceitos conhecidos da cinemática e da mecânica celeste, foi possível obter a velocidade de deslocamento de um planeta ao redor do astro em função do comprimento da trajetória e do tempo que leva para dar uma volta em torno do Sol, além da velocidade máxima em função da excentricidade e do semieixo maior (Tabela 3). Os resultados obtidos usando essa metodologia estão em boa concordância com os dados existentes na literatura.

A ordem dos planetas nas tabelas segue a orientação do planeta mais próximo ao Sol ao mais distante (de cima para baixo), levando em conta o nosso sistema solar, onde se pode notar que à medida que a órbita planetária é de maior comprimento a velocidade vai diminuindo, conforme pode ser visto na Tabela 3. 
Tabela 3 - Tempo de translação e velocidade orbital.

\begin{tabular}{cccccc}
\hline $\begin{array}{c}\text { Corpos } \\
\text { Celestes }\end{array}$ & $\begin{array}{c}\text { Tempo de } \\
\text { translação } \\
\text { (dias) }\end{array}$ & $\begin{array}{c}\text { Velocidade } \\
\text { média } \\
v=\frac{\Delta s}{\Delta t}\end{array}$ & $\begin{array}{c}\text { Velocidade } \\
\text { Média } \\
\text { (Nasa) }\end{array}$ & $\begin{array}{c}\text { V. Orbital } \\
\text { máxima }\end{array}$ & $\begin{array}{c}\text { Velocidade } \\
\text { Orbital }\end{array}$ \\
Vênus - Sol & 224,69 & 35,02 & 35,02 & $\frac{G(1+e)}{a(1-e)}$ & $\begin{array}{c}\text { máxima } \\
\text { (km/s) } \\
\text { (NASA }\end{array}$ \\
Terra - Sol & 365,26 & 29,78 & 29,78 & 35,25 & 35,26 \\
Marte - Sol & 686,98 & 24,07 & 24,07 & 26,28 & 30,29 \\
Júpiter - Sol & $4.332,59$ & 13,06 & 13,06 & 13,70 & 26,50 \\
Saturno - Sol & $10.759,22$ & 9,68 & 9,68 & 10,18 & 13,72 \\
\hline
\end{tabular}

Fonte: NASA (2019).

\section{Conclusões}

Com conceitos básicos das Leis de Kepler, do momento angular de um sistema físico, da conservação de energia total de um corpo, de cinemática elementar e de conhecimentos acerca da resolução de certas integrais elípticas, foi possível calcular a velocidade orbital média de cada corpo celeste selecionado para esta pesquisa. Além disso, também foram adaptadas as expressões para a velocidade orbital mínima e máxima de um corpo planetário em torno do Sol, considerando o nosso sistema solar, e foram obtidas em função dos parâmetros relativos ao eixo maior e a excentricidade elíptica das trajetórias envolvidas. Os resultados obtidos com essa metodologia simples ficaram em boa concordância com os dados coletados na literatura.

As expressões matemáticas da velocidade mínima e velocidade máxima de um corpo celeste dependem dos valores da excentricidade da elipse e do semieixo maior.

\section{Referências}

ALRIGA. Cálculo de la velocidad en órbitas elípticas. Disponível em:

https://forum.lawebdefisica.com/entries/618-C\%C3\%A1lculo-de-la-velocidad-en-\%C3\%B3rbitasel\%C3\%ADpticas. Acesso em: 12 jan. 2019.

ASIMOV, Isaac. 111 Questões sobre a Terra e o Espaço. São Paulo: Círculo do livro,1991.

CANALLE, J. B. G. O problema do ensino da órbita da Terra. Física na Escola, v. 4, n. 2, p. 12-16, 2003. Disponível em: http://www.sbfisica.org.br/fne/Vol4/Num2/v4n2a06.pdf. Acesso em: set. 2019.

COSTA, J. R. V. Os astrônomos. Astronomia no Zênite: o Universo é tudo para nós. jan. 2006. Disponível em: http://www.zenite.nu/os-astronomos/. Acesso em: 22 jun. 2018.

ESQUEF, P. A.; RIBEIRO, S. A. P. Elipses e as órbitas dos planetas. Revista do Professor de Matemática. São Paulo, n. 77, p. 28-32, 2012. Disponível em: http://rpm.org.br/cdrpm/77/7.html. Acesso em: dez. 2017.

GUIDORIZZI, H. L. Um curso de cálculo. v. 2. Rio de Janeiro: LTC, 2002.

HALLIDAY, D.; RESNICK, R.; WALKER, J. Fundamentos de Física, v. 1: Mecânica. 6. ed. Rio de Janeiro: LTC, 2002. 
HASSER, N.; LA SALLE, J.; SULLIVAN, J. Análisis Matemático 1: Curso introductório. México: Trillas, 1990.

LONGHINI, M. D. Será o Cruzeiro do Sul uma cruz? Um novo olhar sobre as constelações e seu significado. Física na Escola, Uberlândia, MG, v. 10, n. 1. p. 26-29, 2009.

MENDONÇA, B. Entrevista concedida à equipe do Globo Ciência. Entenda como funciona o telescópio e sua evolução ao longo dos séculos. 2011. Disponível em:

http://redeglobo.globo.com/globociencia/noticia/2011/11/entenda-como-funciona-o-telescopio-esua-evolucao-ao-longo-dos-seculos.html. Acesso em: 22 jun. 2018.

NASA. National Artonautics and Space Administration. Planetary Fact Sheet. Disponível em: https://nssdc.gsfc.nasa.gov/planetary/planetfact.html. Acesso em: 02 jan. 2019.

NOGUEIRA, S.; CANALLE, J. B. G. Astronomia: Ensino Fundamental e Médio. Coleção explorando o ensino. Brasília: MEC, SEB, MCT, AEB, v. 11, p. 25-46, 2009.

RAMOS. M. Aprenda a se orientar pelos astros. 2006. Disponível em: http://www.invivo.fiocruz.br/cgi/cgilua.exe/sys/start.htm?infoid=800\&sid=3\&tpl=printerview. Acesso em: 01 jan. 2019.

SATO, P. Quais foram as descobertas mais importantes do Hubble? 2009. Disponível em: www.nova.escola.org.br/conteudo/1129/quais-foram-as-descobertas-mais-importantes-do-hubble. Acesso em: 25 jun. 2019.

SILVA, R. B. As 12 mentes mais brilhantes da História da Astronomia. Universo Racionalista. 2016. Disponível em: https://universoracionalista.org/as-doze-mentes-mais-brilhantes-da-historiada-astronomial. Acesso em: 25 jun. 2019.

SILVA, Wilson. Movimento Orbital. In: PRADO, A. F. (Org.). Fundamentos da tecnologia espacial. São José dos Campos: INPE, p. 9-24, 2001.

SIMÕES, C. Unidade Astronômica. Astronoo. 2013. Disponível em: http://www.astronoo.com/pt/artigos/unidade-astronomica.html. Acesso em: 21 jul. 2019.

VENERO, A. Introducción al Analisis Matemático. Lima-Peru: Gemar, 1995.

WILLIAMS, M. What is the closest planet to Earth? Universe Today. 2016. Disponível em: https://www.universetoday.com/14447/what-is-the-closest-planet-to-earth/. Acesso em: 25 mar. 2019. 